\title{
ESTUDIO SOBRE LA MOTIVACIÓN Y LA SATISFACCIÓN LABORAL EN DOCENTES DE LA FACULTAD DE ENFERMERÍA - APLICACIÓN DE LA ESCALA GENERAL DE WARR, COOK Y WALL PARA SU EVALUACIÓN ${ }^{38}$
}

\author{
Mg. Norma Elena Jaime Piñas ${ }^{39}$
}

Facultad de Enfermería de la Universidad Nacional del Centro del Perú

\begin{abstract}
RESUMEN
En el presente estudio fue en la Facultad de Enfermería, se determinaron el nivel de motivación y la categoría de satisfacción laboral de los docentes, y se describió entre sí los resultados obtenidos de distinta categoría laboral. El estudio fue descriptivo de corte transversal, participaron 28 de 33 docentes nombrados, con disposición de participar en la investigación. Las Técnicas y procedimientos de recolección de datos se centraron en la aplicación de dos escalas auto aplicativas, la de motivación con preguntas de respuestas cerradas y la de satisfacción general, la que se subdividió en dos dimensiones cuya valoración se realizó categorizándolo en satisfacción general, satisfacción intrínseca y satisfacción extrínseca. Para el análisis e interpretación se utilizó la estadística descriptiva y para el vaciado de datos el programa Estadístico SPSS versión 15. Los resultados obtenidos confirman que el total de docentes estudiado están motivados de alguna manera, oscilando el nivel de motivación entre muy motivado $(71,4 \%)$ de docentes y medianamente motivados el $28,6 \%$ de docentes, los resultados de docentes que están significativamente muy motivados por categorías es como sigue: Asociados $(73,7 \%$ ), principales $100 \%$ y jefes de práctica $60 \%$, medianamente motivados: auxiliares $100 \%$, jefes de práctica $40 \%$ y asociados $26,3 \%$, evidenciando un funcionamiento promedio sin llegar a presentar niveles óptimos de motivación. Respecto a la categoría de satisfacción laboral la mayoría $(57,1 \%)$ tienen satisfacción laboral y satisfacción intrínseca, y sólo el $50 \%$ de los docentes tienen satisfacción extrínseca, la diferencia muestra docentes que están insatisfechos. Los resultados de las diferentes categorías muestran que la totalidad de docentes principales y auxiliares están satisfechos laboralmente, el $47,4 \%$ de los docentes asociados están satisfechos y la diferencia $52,6 \%$ de docentes de esta categoría no lo están, el $40 \%$ de jefes de práctica tienen satisfacción laboral y el $60 \%$ está insatisfecho. En la satisfacción laboral de los docentes predomina significativamente la satisfacción intrínseca más que la extrínseca. Las características de motivación y de satisfacción laboral detectadas, hace inobjetable la necesidad de optimizarlas con una debida orientación hacia las metas, atendiendo las fortalezas del grupo y de la institución. Los resultados arrojan, razones más que suficientes para establecer estrategias de gestión relacionadas a fortalecer la satisfacción laboral de los docentes, dado el porcentaje significativo de docentes insatisfechos e impulsar mecanismos que alimenten la motivación existente direccionado de la organización hacia el trabajador que son aspectos débiles que deberán ser respaldadas a través de políticas institucionales establecidas para tal fin.
\end{abstract}

Palabras Clave: Motivación docente, satisfacción laboral, satisfacción intrínseca, satisfacción, extrínseca, niveles, categorías.

\section{STUDY ON MOTIVATION AND JOB SATISFACTION IN PROFESSORS OF THE FACULTY OF NURSING IMPLEMENTATION OF THE GENERAL LEVEL OF WARR, COOK AND WALL FOR EVALUATION}

\begin{abstract}
This study was in the Faculty of Nursing, were determined level of motivation and level of job satisfaction of professors, and described each other the results of job category. The study was cross sectional, involving 28 of 33 teachers nominated, with willingness to participate in research. Techniques and procedures for data collection focused on the implementation of selfapplied two scales, the motivation to closed-ended questions and overall satisfaction, which was subdivided into two dimensions whose assessment was categorized into general satisfaction, satisfaction intrinsic and extrinsic satisfaction. For the analysis and interpretation used descriptive statistics and data to empty the SPSS version 15 . The results confirm that the total number of professors studied are motivated in some way, varying the level of motivation among highly motivated (71.4\%)

38

Trabajo de investigación fue recibido el 07/05/2010 retornado para su revisión el 15/06/2010 y aprobado para su publicación 16/11/2010.

39

Mg. En Enfermeria E-mail: normajaimep@yahoo.es
\end{abstract}


of professors and $28.6 \%$ moderately motivated professors, educational outcomes are significantly motivated by categories as follows: Associated (73.7\%), Principals $100 \%$ and $60 \%$ practice chief, moderately motivated, auxiliary $100 \%, 40 \%$ practice chiefs and associated $26.3 \%$, showing an average performance without present reach optimum levels of motivation. Regarding the category of job satisfaction most (57.1\%) have job satisfaction and intrinsic satisfaction, and only $50 \%$ of professors have extrinsic satisfaction, the difference shows professors who are dissatisfied. The results of the different categories show that all main and auxiliary professors are paid labor, $47.4 \%$ of professors partners are satisfied and the difference $52.6 \%$ of professors in this category are not, $40 \%$ practice managers have job satisfaction and $60 \%$ are dissatisfied. In the professors' job satisfaction significantly dominates the intrinsic satisfaction rather than extrinsic. The characteristics of motivation and job satisfaction detected, makes the need to optimize unobjectionable with proper guidance toward goals, taking the strengths of the group and the institution. The results show, more than enough reason to establish management strategies related to strengthening the professors' job satisfaction, given the significant percentage of dissatisfied professors and promote mechanisms that fuel the motivation of the organization directed toward the worker who is weak, aspects should be supported through institutional policies established for that purpose.

Key words: Professor, motivation, job satisfaction, intrinsic satisfaction, extrinsic satisfaction, level categories.

\section{INTRODUCCIÓN}

Es un reto para toda Institución no solo mantener a las personas en la organización, trabajando y dando el máximo de sí, con actitud positiva y favorable, punto clave para gestionar la interacción entre las aptitudes, intereses, necesidades, motivación, talento, expectativas, hábitos y valores del ser humano con la cantidad y calidad de resultados, sino también lograr la satisfacción que la persona pueda conseguir de su trabajo.

La motivación al actuar como fuerza impulsora de la conducta humana, es un elemento de importancia en cualquier ámbito de la vida, pero cobra un especial valor en el trabajo ya que es en esta actividad que ocupamos gran parte de nuestra existencia, por lo que es necesario estar motivados por ella de modo que no se convierta en una actividad alienada y opresora; sino más bien en un medio que nos produzca satisfacción.

La satisfacción laboral de los trabajadores, puede considerarse como un fin en sí misma, que compete tanto al trabajador como a la organización; que además de producir beneficios a los trabajadores al ayudarles a mantener una buena salud mental, puede contribuir a mejorar la productividad de una organización ya que un trabajador motivado y satisfecho está en mejores condiciones de desempeñar un trabajo adecuado, que otro que no lo esté.

Dentro de este marco la Universidad como organización educativa formadora de recursos humanos profesionales, reconoce la importancia de los docentes en el logro de los objetivos y metas de los procesos educativos considerándolos como recursos estratégicos, por tanto una de las condiciones de trabajo del docente de la Facultad de Enfermería, debe ser su compromiso profesional con la Institución la que debe ser fomentada por la organización ofreciéndole las condiciones necesarias que influirá en la determinación de la calidad educativa, puesto que un docente no motivado e insatisfecho no tendrá el mismo rendimiento en su labor, "perderá" horas de clase en caso de absentismo, y su falta de identificación con la misión y visión de la Universidad y Facultad será evidente, lo que repercutirá muy negativamente en la formación de recursos humanos profesionales.

Es por ello que el presente trabajo de investigación estudió al docente de la Facultad de Enfermería en atención a la búsqueda constante de asegurar la mejora de la gestión, de la calidad humana profesional, de su relación con el entorno y su contribución con las metas Institucionales la que consideramos clave para el desarrollo organizacional de nuestra Facultad.

\section{MATERIALES Y MÉTODOS}

El estudio fue descriptivo de corte transversal. Aplicándose el método científico como elemento central de la investigación. El área de trabajo fue la Facultad de Enfermería, la Población: sus docentes, participaron 28 de 33 Profesionales nombrados de distinta categoría laboral adscritos a la Facultad, que tuvieron la disposición de participar en la investigación. Para determinar los niveles de motivación y las categorías de satisfacción laboral en la población de estudio se aplicó dos escalas auto aplicativas.

La escala de motivación con preguntas de respuestas cerradas las que fueron contestadas por ellos (as) de acuerdo a la frecuencia con que éstas afirmaciones se dieron en los docentes y la escala de satisfacción general, la que se subdividió en tres dimensiones cuya valoración se realizó categorizándolo en satisfacción general, satisfacción intrínseca y satisfacción extrínseca. Para procesar las respuestas se realizó el procesamiento de las mismas, las que fueron ingresadas en el programa Excel. La información se analizó e interpretó utilizando la estadística 
descriptiva y para el procesamiento de datos el programa Estadístico SPSS versión 15.

\section{RESULTADOS}

\section{NIVELES DE MOTIVACIÓN DE LOS DOCENTES}

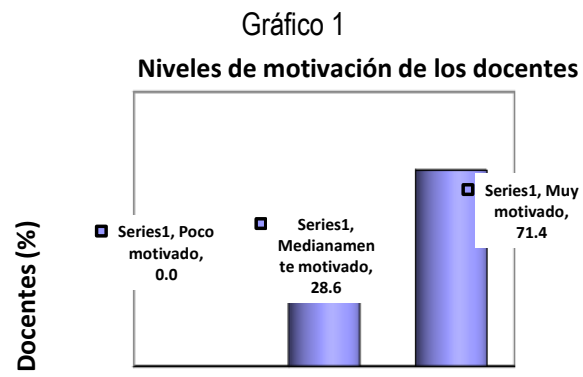

Niveles de motivación

Fuente: Encuesta aplicada por la investigadora.

En el gráfico 1 se aprecia que la mayoría de docentes $(71,4 \%)$ de la Facultad de Enfermería de la UNCP están muy motivados y el $28,6 \%$ lo están medianamente motivados. No hay ningún docente poco motivado.

Tabla 1

Niveles de motivación, según categoría docente

\begin{tabular}{|c|c|c|c|c|c|c|}
\hline \multirow{3}{*}{$\begin{array}{c}\text { Categoría } \\
\text { docente }\end{array}$} & \multicolumn{4}{|c|}{ Niveles de motivación } & \multirow{2}{*}{\multicolumn{2}{|c|}{ Total }} \\
\hline & \multicolumn{2}{|c|}{ Med. Motivado } & \multicolumn{2}{|c|}{ Muy motivado } & & \\
\hline & $\mathrm{F}$ & $\%$ & $\mathrm{~F}$ & $\%$ & $f$ & $\%$ \\
\hline $\begin{array}{l}\text { Jefe de } \\
\text { prácticas }\end{array}$ & 2 & 40,0 & 3 & 60,0 & 5 & 14,3 \\
\hline Auxiliar & 1 & 100,0 & & & 1 & 3,6 \\
\hline Asociado & 5 & 26,3 & 14 & 73,7 & 19 & 67,9 \\
\hline Principal & & & 3 & 100,0 & 3 & 10,7 \\
\hline Total & 8 & 28,6 & 20 & 71,4 & 28 & 100,0 \\
\hline
\end{tabular}

Fuente: Encuesta aplicada por la investigadora.

En la tabla 1 se observa que la mayoría de docentes son Asociados (67,9\%), el 10,7\% Principales, el 3,6\% Auxiliares y el $14,3 \%$ Jefes de prácticas. La totalidad de docentes principales y la mayoría de docentes asociados $(73,7 \%)$ y jefes de prácticas $(60 \%)$ están muy motivados, mientras que la totalidad de docentes auxiliares están medianamente motivados.

\section{Categoría de satisfacción laboral de los docentes Gráfico 2}

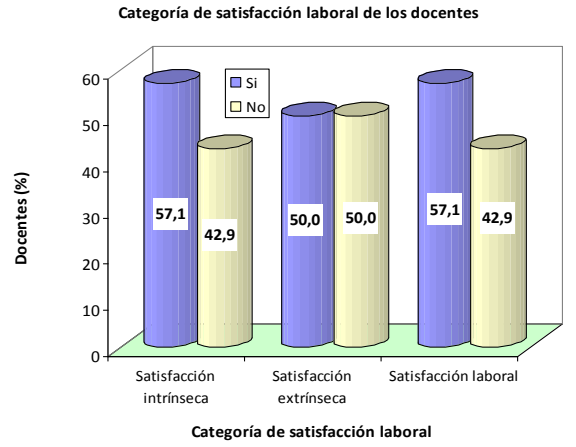

Fuente: Encuesta aplicada por la investigadora.

En el gráfico 2 se constata que la mayoría de los docentes $(57,1 \%)$ tienen satisfacción laboral y satisfacción intrínseca, en tanto que sólo el $50 \%$ de los docentes tienen satisfacción extrínseca.

Tabla 2

Categoria de satisfacción laboral, según categoría docente

\begin{tabular}{|c|c|c|c|c|c|c|c|c|c|c|}
\hline \multirow{3}{*}{$\begin{array}{c}\text { Satisfacción } \\
\text { intrinseca }\end{array}$} & \multicolumn{8}{|c|}{ Categoría docente } & \multirow{2}{*}{\multicolumn{2}{|c|}{ Total }} \\
\hline & \multicolumn{2}{|c|}{ Jefe de $P$. } & \multicolumn{2}{|c|}{ Auxiliar } & \multicolumn{2}{|c|}{ Asociado } & \multicolumn{2}{|c|}{ Principal } & & \\
\hline & Si & No & $\mathrm{Si}$ & No & $\mathrm{Si}$ & No & $\mathrm{Si}$ & No & $\mathrm{Si}$ & No \\
\hline \multirow{3}{*}{$\begin{array}{l}\text { Satisfacción } \\
\text { intrínseca }\end{array}$} & 2 & 3 & 1 & & 10 & 9 & 3 & & 16 & 12 \\
\hline & 40 & 60 & 10 & & 52 & 47 , & 10 & & 57 & $42,9 \%$ \\
\hline & $\%$ & $\%$ & $0 \%$ & & $6 \%$ & $4 \%$ & $0 \%$ & & $1 \%$ & \\
\hline \multirow{3}{*}{$\begin{array}{l}\text { Satisfacción } \\
\text { extrinseca }\end{array}$} & 2 & 3 & 1 & & 8 & 11 & 3 & & 14 & 14 \\
\hline & 40 & 60 & 10 & & 42 & 57, & 10 & & 50 & $50 \%$ \\
\hline & $\%$ & $\%$ & $0 \%$ & & $1 \%$ & $9 \%$ & $0 \%$ & & $\%$ & \\
\hline \multirow{2}{*}{$\begin{array}{l}\text { Satisfacción } \\
\text { laboral }\end{array}$} & 2 & 3 & 1 & & 9 & 10 & 3 & & 15 & 13 \\
\hline & $\begin{array}{l}40 \\
\%\end{array}$ & $\begin{array}{l}60 \\
\%\end{array}$ & $\begin{array}{l}10 \\
0 \%\end{array}$ & & $\begin{array}{l}47, \\
4 \%\end{array}$ & 52, & 10 & & $\begin{array}{l}57, \\
1 \%\end{array}$ & $42,9 \%$ \\
\hline
\end{tabular}

Fuente: Encuesta aplicada por la investigadora.

En la tabla 2 se descubre que la totalidad de docentes principales y auxiliares están satisfechos laboralmente y sólo el $47,4 \%$ de los docentes asociados y el $40 \%$ de los jefes de prácticas tienen satisfacción laboral. Asimismo, la totalidad de docentes principales y auxiliares, la mayoría de los docentes asociados $(52,6 \%$ ) y sólo el $40 \%$ de los jefes de prácticas tienen satisfacción intrínseca. De igual modo, la totalidad de docentes principales y auxiliares sólo el 42,1\% de los docentes asociados y el $40 \%$ de los jefes de prácticas tienen satisfacción extrínseca.

\section{DISCUSIÓN}

La Facultad de Enfermería es una organización formadora de Recursos Humanos, su plana docente la componen profesionales de distintas disciplinas con mayor predominio de profesionales en Enfermería de género femenino, mayores de edad casados en su mayoría y con buena relación de pareja en términos generales, con hijos entre uno y dos de quienes actualmente también son 
responsables directos. En el ámbito estrictamente laboral, los docentes están actualizados ya que la mayoría tiene estudios de maestría y doctorados y en un número significativo ya poseen el grado, están posicionados en las categorías más altas dentro de sistema Universitario, dedicados en su mayoría exclusivamente a la carrera docente con amplia experiencia y muchos años de servicio docente. Algunos de ellos ocupan cargos administrativos de importancia para la gestión de la Facultad y la mayoría de ellos se dedican exclusivamente a la docencia.

Por los resultados obtenidos se pone en relieve que la motivación en los docentes de la Facultad de Enfermería está presente en todos los docentes estudiados, si bien es cierto su presencia no tiene un comportamiento idéntico en cada uno de ellos pero es evidente que se manifiesta. Cada docente tiene particularidades individuales que va a marcar diferencias en las reacciones frente a la sensación de estar motivado 0 a los estímulos que la activen lo que se sustenta en la teoría de las atribuciones de Kelley, 1967 denominado expectativa o instrumentalidad de la motivación de acuerdo a la fuente del refuerzo para el trabajo: motivación extrínseca e intrínseca. En la motivación extrínseca, el empleado muestra conductas de trabajo atribuibles a resultados derivados de fuentes diferentes del trabajo mismo como los compañeros de trabajo, supervisor del empleado o la organización misma. En un estado de motivación intrínseca, el empleado muestra comportamientos de trabajo atribuibles a resultados derivados del trabajo mismo, en general, se asocia a la necesidad de completar las propias potencialidades, el deseo de las personas por investigar, explorar y dominar su entorno y la importancia para el individuo de triunfar en tareas desafiantes y en asumir responsabilidades, las personas dicen estar más intrínsecamente motivadas cuando se perciben a sí mismas como la fuente de su comportamiento. Significaría entonces que la motivación que se percibe en el docente de la FEN se atribuiría más a lo segundo es decir a lo intrínseco personal profesional que al entorno 0 a aspectos de gestión.

Si bien es cierto queda confirmado que la motivación está presente en los docentes de la Facultad de Enfermería en sus dos más altos niveles, lo que preocuparía sería que esta motivación percibida no sea solo desde los trabajadores sino más bien complementado con la motivación extrínseca desde la organización, para que no sea solo una sensación eventual sino más bien permanente y sobre todo sostenible que tendría que ver más con las estrategias aplicadas desde una proyección moderna de gestión y en este aspecto coincidimos con Moreno (1997), en que "es la organización la que más facilita la integración de funciones y su orientación al proceso global, lo cual permite a cada actor, entender y evaluar su aportación al conjunto". Se trataría entonces de potenciar la motivación extrínseca, referidos al entorno del docente dentro de la Facultad y cuando ésta sea altamente satisfactoria, se autoalimentaría para una duración indefinida. Si se consigue esta motivación en la organización, se habrán sentado las bases para que ésta aprenda y se desarrolle, de lo contrario el riesgo sería no solo para el docente que puede dejar de estar motivado si no para la organización, considerando a la desmotivación como uno de los principales causantes de bajo rendimiento laboral absentismo, falta de identificación Institucional etc. que repercutiría en la calidad académica. Por ello, se ha de prestar especial atención a este aspecto y su comportamiento en el colectivo profesional de la Facultad ya que se espera que el conocimiento que se pueda generar sirva para ejercer una labor preventiva sobre este tema.

Por otro lado los resultados muestran presencia de satisfacción docente en un porcentaje resaltante sin embargo, nuestra atención se focaliza en el porcentaje significativo de docentes que manifiestan no estar satisfechos laboralmente con una ligera tendencia mayoritaria a la no satisfacción extrínseca más que a la intrínseca específicamente en la categoría de asociados, ello implica que los docentes sienten descontento con aspectos relativos a la organización del trabajo, a los horarios, a la remuneración, a las condiciones físicas del trabajo entre otros es decir al entorno laboral y en segundo plano a los aspectos relacionados al reconocimiento obtenido por el trabajo, la responsabilidad, la promoción laboral, al contenido de la tarea etc.,

Una de las teorías que más ha influido en el área de la satisfacción laboral es la denominada teoría de los dos factores 0 teoría bifactorial de la satisfacción de Herzberg. Herzberg postuló la existencia de dos grupos o clases de aspectos laborales: un grupo de factores extrínsecos y otro de factores intrínsecos(personal/profesional), los primeros están referidos a las condiciones de trabajo en el sentido más amplio, tales como el salario, las políticas de la organización, el entorno físico, la seguridad en el trabajo, etc. Esta teoría explicaría porque estando los docentes motivados dentro de la organización hay docentes insatisfechos una primera explicación radicaría en las particularidades propias del docente, y la segunda más contundente porque contrastando los resultados tanto de motivación como de satisfacción laboral probablemente los docentes motivados por factores intrínsecos estén también satisfechos por factores intrínsecos es decir más a lo personal/ profesional y trabajo en sí muy desligados con otros aspectos del entorno como, ambiente laboral facilidades institucionales, gestión etc. Por ello el modelo planteado por Herzberg señala que la satisfacción laboral 
sólo puede venir generada por los factores intrínsecos (a los que Herzberg llamó "factores motivadores") mientras que la insatisfacción laboral sería generada por los factores extrínsecos (a los que Herzberg dio la denominación de "factores higiénicos") que es lo que al parecer estaría sucediendo en este caso. Teoría que sustentaría los resultados obtenidos en el tema de satisfacción laboral y explicaría la presencia muy significativa de docentes de distintas categorías laborales que estando motivados estén insatisfechos en la Facultad. Según el modelo bifactorial estos factores extrínsecos sólo pueden prevenir la insatisfacción laboral o evitarla cuando ésta exista pero no pueden determinar la satisfacción ya que ésta estaría determinada por los factores intrínsecos, que serían aquellos que son consustanciales al trabajo; contenido del mismo, responsabilidad, logro, etc.

Los resultados muestran claramente la carga de insatisfacción general del docente en un porcentaje significativo, que amerita ser absuelto por las autoridades ya que si bien es cierto hay algunas situaciones que escapan de la posibilidad de atención, existen otros que si son situaciones factibles de atender con una adecuada toma de decisiones y capacidad de gestión.

De acuerdo con los objetivos de este trabajo, las conclusiones más relevantes a las que podemos llegar es que el nivel de motivación en los docentes de la Facultad de Enfermería, está entre muy motivado $(71,4 \%)$ y medianamente motivados el $28,6 \%$ de docentes. Los resultados obtenidos del grupo principales, asociados auxiliares y jefes de práctica de la Facultad de Enfermería están significativamente muy motivados, el personal docente asociado $(73,7 \%)$, principal $100 \%$ y jefes de práctica $60 \%$, medianamente motivados los auxiliares $100 \%$ jefes de práctica $40 \%$ y asociados $26,3 \%$. La motivación en los docentes es buena, sin llegar a presentar niveles óptimos de motivación, cuyas características está basada en factores intrínsecos (autorrealización personal y profesional). La motivación que presenta el grupo contrasta con los mínimos requerimientos organizacionales que permitan asegurar el logro de metas personales e institucionales que debe caracterizar a instituciones que como éstas giran en torno a la formación humana.

En cuanto se refiere a la jerarquía o categoría de satisfacción laboral en los docentes de la Facultad de Enfermería es que la mayoría de los docentes $(57,1 \%)$ tienen satisfacción laboral y satisfacción intrínseca, en tanto que sólo el $50 \%$ de los docentes tienen satisfacción extrínseca, la diferencia porcentual es de docentes que están insatisfechos. Los resultados obtenidos del grupo de docentes principales, asociados auxiliares y jefes de práctica de la Facultad de Enfermería, la totalidad de docentes principales y auxiliares están satisfechos laboralmente, el $47,4 \%$ de los docentes asociados están satisfechos y la diferencia es decir el $52,6 \%$ de docentes de esta categoría no lo están, el $40 \%$ de los jefes de prácticas tienen satisfacción laboral y el $60 \%$ está insatisfecho. En la satisfacción laboral de los docentes predomina significativamente la satisfacción intrínseca más que la extrínseca.

Las características de motivación y de satisfacción laboral detectadas hace inobjetable la necesidad de optimizarlas con una debida orientación hacia las metas y atendiendo las fortalezas del grupo y de la institución. La concordancia entre el tipo de institución y los niveles de poder constituye un soporte administrativo útil para efectos de intervención organizacional.

Por ser un estudio puramente descriptivo, es imposible establecer relaciones causa-efecto entre las variables, investigaciones futuras habrán de profundizar al respecto. Esperamos que el conocimiento que se pueda generar con este estudio sirva para ejercer acciones institucionales orientadas a mejorar estrategias en la gestión de recursos humanos.

\section{REFERENCIAS BIBLIOGRÁFICAS}

- Ardouin, J., Bustos, C., Gayó, R. \& Jarpa, M. Motivación y Satisfacción Laboral.[En línea] 2000. http://www2.udec.cl/ ivalfaro/apsique/labo/motysat is.html

- González R. La motivación en las Organizaciones: Su reflejo en el sistema empresarial cubano. 2000.

$\checkmark$ http://www.monografias.com/trabajos $16 / \mathrm{m}$ otivacion-organizaciones/motivacionorganizaciones.shtml\#mmotivos

- $\quad$ Salinas,C., Laguna, J. \& Mendoza,MR La satisfacción laboral y su papel en la evaluación de la calidad de la atención médica. Salud Pública Mex; 36:2229.1994. 